\title{
Eye Tracking and Gaze Based Interaction within Immersive Virtual Environments
}

\author{
Adrian Haffegee and Russell Barrow \\ Advanced Computing and Emerging Technologies Centre, \\ The School of Systems Engineering, University of Reading, UK \\ a.haffegee@reading.ac.uk
}

\begin{abstract}
Our eyes are input sensors which provide our brains with streams of visual data. They have evolved to be extremely efficient, and they will constantly dart to-and-fro to rapidly build up a picture of the salient entities in a viewed scene. These actions are almost subconscious. However, they can provide telling signs of how the brain is decoding the visuals, and can indicate emotional responses, prior to the viewer becoming aware of them.

In this paper we discuss a method of tracking a user's eye movements, and use these to calculate their gaze within an immersive virtual environment. We investigate how these gaze patterns can be captured and used to identify viewed virtual objects, and discuss how this can be used as a natural method of interacting with the Virtual Environment. We describe a flexible tool that has been developed to achieve this, and detail initial validating applications that prove the concept.
\end{abstract}

\section{Introduction}

Psychological studies often use eye tracking to gather information relating to how a user reacts to particular visuals. Typical uses would be for areas such as marketing aesthetics, the effectiveness of emergency signage, or measuring attention 12 . Current methods record the gaze position on a $2 \mathrm{D}$ image or video stream, with the captured data being stored for offline analysis.

Virtual Environments (VE) are used to create alternate worlds that users can enter and interact with. These worlds are configurable and controllable, and are well suited for constructing scenes that would be difficult or time consuming to build in the real world e.g., those that are hazardous, dynamic, or too expensive.

By using eye tracking within the $\mathrm{VE}$, it is possible to capture a user's eye movements and analyse how they are observing the scene. Because the composition of the VE is known, these actions can be directly mapped to entities within the scene providing the possibility of automatic analysis of where a user is looking. This could then be used for interaction with the environment itself, with previous work considering sight operated pointing and selecting 34. Although there has been a reasonable research with eye tracking within VEs, there has been little within modern 3D immersive projection technologies. This combination with these systems that provide a more natural interactive experience has 
wide potential. In multiuser environments, research has been undertaken on the importance of gaze to aid communication amongst remote participants [5].

In the next section we will discuss the system configurations that have been used, and why they were chosen. Section 3 will then detail the methods and algorithms used in the implementation. Section 4 will cover the validation and a basic sample application, before Sect. 5 concludes the paper and describes potential future extensions.

\section{System Configuration}

This research focuses on the problem of enabling a head mounted eyetracker to be used inside an Immersive Virtual Environment (IVE). The intelligent coupling of these two systems makes it possible to calculate where in the virtual scene a user is looking. The main components are the immersive VR system, the eyetracker, and software that binds it together.

Immersive Systems. Immersive projection based technologies such as the CAVE 7] place the user in an environment surrounded by one or more projection screens. The user is free to move within the confines of the system. They are position tracked and the perspective of their viewed images are adjusted according to their relative head position. While more expensive than other immersive technologies such as Head Mounted Displays (HMDs), these systems are less restrictive and provide a more natural view of the environment in addition to the methods of interacting with it. These systems provide the user with a higher degree of presence within the VE [8]. This is the degree that they feel that they are a participant within the environment. The greater the feeling is, the more likely they are to instinctively behave and react as though they were within a real situation.

The Mobile Eye. The ASL Mobile Eye eye tracker [9], was used for this project. It is lightweight and glasses mounted, and is well suited for use within IVEs where the user will be free to move around. This is in contrast to freestanding trackers, which require the user to remain relatively still, and at a fixed position from the device. Other head mounted devices could use the approach described, but may need minor modifications depending on the format and structure of their data output.

The Mobile Eye uses Dark Pupil Tracking to calculate gaze position. This uses the pupil position and the reflection from the cornea to determine the eye's direction. Figure 1 shows how we combine the system with a standard head tracker (here an Intersense IS-900), which obtains the user's head position and orientation within the environment. Attached to the glasses are two cameras; one aimed in the direction of the user's vision, and the second capturing an image of their eye reflected by the combiner.

The system is calibrated to provide a gaze position for where the user is looking at any time. This is indicated to the user by overlaying a gaze position marker on top of the output video stream from the scene camera. A sample 

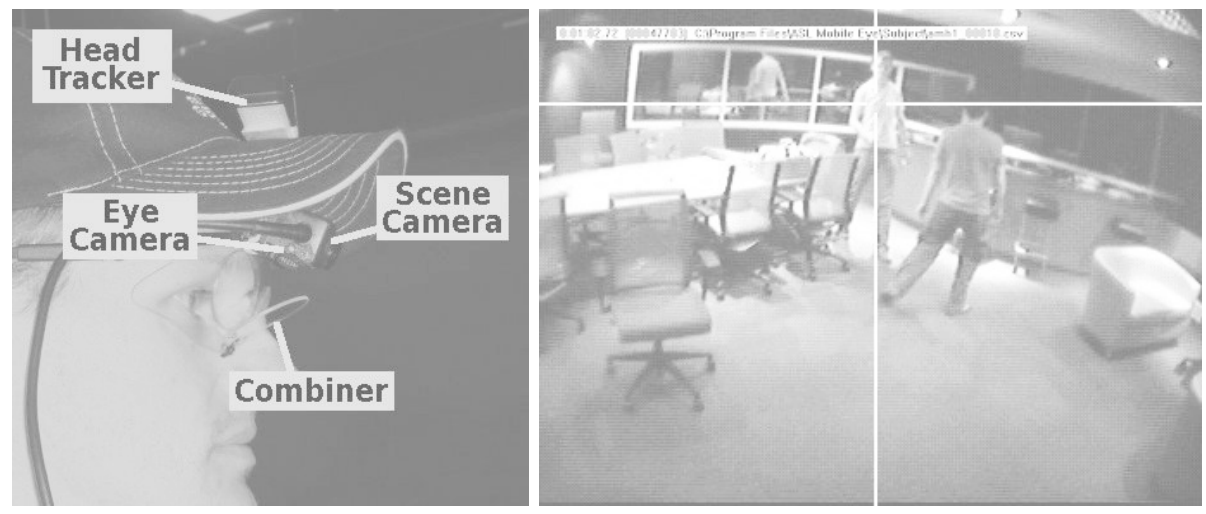

Fig. 1. The ASL Mobile Eye device, showing how it is combined with a head tracker (left). Video output from the device, with a crosshair overlay for gaze direction (right).

output from this can be seen in the right hand image of Fig. 1 Here a crosshair is being used to represent the user's line of sight. The $(\mathrm{x}, \mathrm{y})$ coordinates of the marker as displayed on the video image can optionally be streamed in an encoded format from the analysis computer's serial port. It is these Point of Gaze (PoG) coordinates that we use for calculating our virtual world gaze tracking.

Virtual Environment Application Development. Different methods and tools are available for the creation of IVEs. This research used the VieGen framework 10, which is a set of tools and utilities to aid application development. Entities within the virtual world are represented by members of an extensible family of SceneNodes. These contain the configurable attributes and behaviours of the objects, can be dynamically loaded at runtime, and provide a harness for developers to extend the environment.

\section{Calculating and Using the Gaze Vector}

This project converts the PoG output from the Mobile Eye into a virtual world gaze vector. This is a vector starting at the user's eye position and heading off in the direction of their line of sight. Within the VE, this vector can be used to indicate potential areas of visual interest, or as advanced methods of controlling the environment. Being glasses mounted, the Mobile Eye's frame of reference is that of the head tracker offset by the distance from the tracker to the eye. This relationship provides a method of converting from the (x,y) PoG coordinate output into the $3 \mathrm{D}$ virtual world gaze vector.

Figure 2 shows a breakdown of the modules developed for this research. The left image shows the high level components which have been wrapped into a VieGen Dynamic SceneNode, allowing for rapid development and portability. The Eye Tracking Control Module provides the core functionality from this research, and could easily be extracted for incorporation into any other VR 

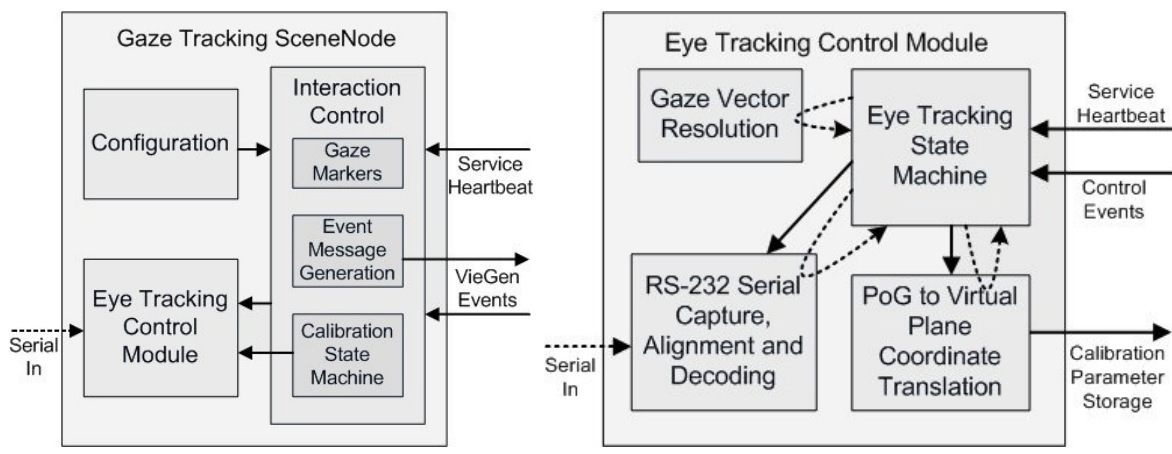

Fig. 2. Gaze Track SceneNode overview (left) \& Eye Tracking Control Module (right)

application or framework. The right image shows the breakdown of this core component, containing the RS-232 (serial) stream decoding, coordinate translation, conversion to gaze vector, and the state machine that binds it together. In addition to the control module the SceneNode also contains configuration data and an interaction component responsible for how the node will react with the rest of the VE.

\subsection{Eye Tracking Control Module}

Serial Capture, Alignment and Decoding. The Mobile Eye streams the encoded tracking information as consecutive 10 byte blocks of serial data. This component locks onto the stream to locate the start of each block, and then decodes the data into a structure which contains the PoG coordinates in the video stream. If the tracker fails to calculate the eye position, (e.g. due to the user blinking or removing the glasses), a status byte within this structure is used to indicate an error condition.

Mapping PoG Coordinates on to a Virtual World Plane. The PoG coordinates can be considered as the $(\mathrm{x}, \mathrm{y})$ coordinates on a plane that is a constant distance and perpendicular to the user's head position. A similar plane can be created in virtual space maintaining a fixed position relative to the user's head tracked location. A relationship between the real and virtual gaze positions can be obtained by having the user fixate on a known point on the virtual plane, while reading the PoG coordinates streamed from the Mobile Eye. The software takes several readings for each of these fixation points, and averages the valid ones to minimise errors or inaccuracies. By sampling a number of these relationships across different positions on the gaze plane, a calibration mapping of PoG $(x, y)$ position to virtual plane location can be constructed.

This calibration data is stored within the module, and can be further analysed to determine its nature. It was observed that for the Mobile Eye there was a linear correlation between the PoG coordinates and the virtual plane positions. However it should be noted that different cameras/lenses could deviate from this 
and would require subsequent algorithm modifications. Ideally future versions would be able to automatically self-check the calibration data and could prompt for recalibration if required.

From the calibration mapping it is desirable to formulate a method of calculating the virtual gaze plane interception location from any PoG $(\mathrm{x}, \mathrm{y})$ position. Assuming that the mapping is linear the gaze plane location can be calculated by comparing the unknown position's PoG value relative to two of the known calibration points. Ideally the chosen points should be sufficiently distant from each other to reduce the effect of errors in the calibration data. The reliability of this calculation can then be further improved by combining the results obtained relative to a number of these calibration point pairs. However small degrees of non-linearity in the mapping would introduce errors the greater the distance to these calibration points.

Obtaining the Gaze Vector. The virtual gaze plane is located at a fixed offset from the user's head, the location of which is known within the VE. By applying the eye offset to the head location the eye position can be found. The gaze vector is a ray starting at the eye and heading through the gaze plane point of interception, and off into the distance. This ray can be applied to the virtual scene to determine the first object that it intercepts. Assuming the object is visible it will be the object being viewed.

\subsection{Scene Interaction Control}

The gaze tracking functionality is wrapped in a VieGen SceneNode, which provides useful infrastructure for interfacing with the virtual scene. It includes the functionality for calculating the gaze vector, and also a state machine for controlling the calibration process, markers for indicating the direction or position of gaze, and event message handling for informing other scene objects if they are being viewed. Although this section is based on the VieGen infrastructure, the methods and algorithms could be ported to other VE development frameworks.

Calibration. Although the PoG calibration mapping is handled in the eye tracking control module, the process of conducting a calibration run is controlled by the SceneNode. The user is required to sequentially fixate on known points distributed about the virtual gaze plane. A state machine manages this process, displaying the gaze plane in the $\mathrm{VE}$ and using a sphere to indicate where the user should be looking. A green sphere is used to indicate reception of valid readings from the mobile eye, and this is changed to red should they switch to invalid. During the sampling process for each sphere position, the gaze plane changes red to inform the user that they need to remain fixated. Once the background returns to grey the sampling will have finished, and the sphere will either move to the next location or will remain in place should it need to be repeated.

Upon completion of all calibration points the system stores the set of calibration data. A simple graphing object within the VE was used to represent the mappings for each of the $\mathrm{X}$ and $\mathrm{Y}$ coordinates. The left image of Fig. 3 shows 

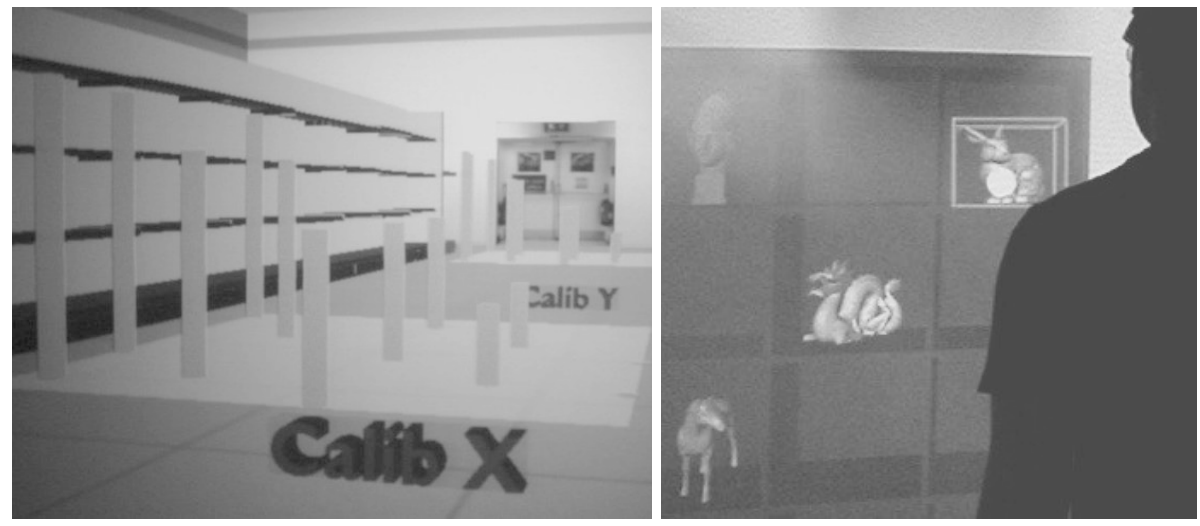

Fig. 3. In-scene visualization of calibration data to validate results (left), \& live gaze selection in the virtual gallery, with multiple indicators for the viewed object (right)

a typical output from these graphs for the Mobile Eye. As can be seen, these should display a linear mapping. The provision of graphs that can be viewed within the VE enables in-scene indication of the reliability of the calibration data. This simplifies the process of identifying inaccuracies in either individual calibration points or the complete set.

Gaze Position Markers, and Message Events. A simple way of indicating the gazed object is by adding a marker to the scene at the gaze vector intersection point, however this may not be the best approach. If there is any error from the calibration the marker may be slightly offset from the viewed position. Naturally the users eyes will be drawn to the marker, resulting in a new position for the marker, again slightly offset. This repeats, resulting in a marker that appears to wander the scene. The involuntary eye action can wrongly lead to assumptions about the system stability. This can be resolved by using different markers. One such approach is to use a bounding box around the object being viewed. This provides a more stable indication, but can lose the accuracy as to which part of the object was being viewed. If the scene objects have been named, their textual information can be displayed within the scene, but again this does not convey the exact hit location. An alternative method is not to display the marker in the user's view of the scene, but to store a log of its position for separate display to interested third parties.

Some VEs allow virtual objects to create and consume message events. For VieGen, an event has been defined to indicate that an object is being viewed, and this is forwarded to the first object intersected by the gaze vector. While current reactions to this event are limited, future responses could include moving, animating, or other methods of interaction between the object and the viewer.

Configuration. For this project, the gaze tracking SceneNode can be configured at run time. It uses XML to define the com port to be used for the serial 
connection between the analysis computer and the VR system, the offset from the head tracker to the users eye, the position and size of the virtual gaze plane, and the number and locations of calibration points used in the PoG mapping.

\section{Validation and Virtual Gallery Application}

The practical experiences during the development of this technology have clearly shown that the immersive eye tracking developed has been successful. The basic functionality of the gaze tracking SceneNode enables a gaze marker to be displayed which indicates the location in the environment that the user is viewing. To further prove the features of the technology an eye gaze specific application was developed based around a virtual gallery. This VE consists of a display case containing various artifacts that the user can view. It has been enhanced with in-scene menus to start calibration, add or remove different types of indication markers, and to start/stop the logging to disk of the gaze vector interception positions. The latter of these can be used for subsequent analysis and replay of the user's viewing patterns. The photo on the right of Fig. 3 shows this application in use. The user is able to select the different objects solely by using their eyes, with movements of their head and body having no detrimental effect on the selection process. In this example, a sphere is used as a gazed object marker along with a wireframe bounding box, and these can be seen on the selected rabbit. During the tests, the gaze positions were recorded and these could be replayed within the scene to show the users viewing patterns.

\section{$5 \quad$ Summary and Future Work}

While no formal evaluation tests have yet been conducted with this research, the initial results clearly demonstrate its feasibility. Once calibrated the system will reliably follow the users eye position regardless of how they move both in the virtual world and within the confines of the VR system.

However there is still scope for optimization of the algorithms used, particularly in the field of calibration. The relationship between the PoG and the gaze plane should be further studied to determine the nature of this relationship and to investigate if can be represented mathematically rather than as a comparison between calibration points. It would also be useful if the different calibration points could be compared to assess their reliability and accuracy. A further extension could involve modifications to the Mobile Eye analysis software which would allow it to receive scene coordinates from the VR system. It this case the complete system could be calibrated inside the VE, and this would do away with the need for the intermediate gaze plane currently being used.

Additional extensions could also be developed to aid analysis of the captured user data. In addition to walk-throughs of the VE that replay dynamic user head and eye positioning, these could also include hot-spots indicating areas of particular interest. Analysts could explore and navigate these VEs, with superimposed markers demonstrating the viewing behaviour. Indeed, multiple user input files 
could be combined to allow more quantitative analysis. These could be filtered as desired, and overlaid on the scene to show key areas of interest.

There is vast potential in the use of this technology, and many researchers may benefit from studying eye behaviour from within a fully controllable environment. Commercially this could include market research or safety analysis, where attracting a user's attention visually is important. Research could include how eye movements are used as communication extensions. By extending the technology as a control or navigation interface, it could also provide a natural method of interaction. This could be especially useful for those with disabilities that preclude them from otherwise controlling or participating within the VE.

\section{References}

1. Duchowski, A.T.: Eye Tracking Methodology: Theory and Practice. Springer, New York (2007)

2. Cox, A.L., Cairns, P., Berthouze, N., Jennett, C.: The use of eyetracking for measuring immersion. In: CogSci 2006 Workshop: What have eye movements told us so far, and what is next?, Vancouver, Canada (July 2006)

3. Tanriverdi, V., Jacob, R.J.K.: Interacting with eye movements in virtual environments. In: CHI, pp. 265-272 (2000)

4. Asai, K., Osawa, N., Takahashi, H., Sugimoto, Y.Y., Yamazaki, S., Samejima, M., Tanimae, T.: Eye mark pointer in immersive projection display. In: VR 2000: Proceedings of the IEEE Virtual Reality 2000 Conference, Washington, DC, USA, p. 125. IEEE Computer Society, Los Alamitos (2000)

5. Garau, M., Slater, M., Vinayagamoorthy, V., Brogni, A., Steed, A., Sasse, M.A.: The impact of avatar realism and eye gaze control on perceived quality of communication in a shared immersive virtual environment. In: CHI 2003: Proceedings of the SIGCHI conference on Human factors in computing systems, pp. 529-536. ACM, New York (2003)

6. Murray, N., Roberts, D., Steed, A., Sharkey, P., Dickerson, P., Rae, J.: An assessment of eye-gaze potential within immersive virtual environments. ACM Trans. Multimedia Comput. Commun. Appl. 3(4), 1-17 (2007)

7. Cruz-Neira, C., Sandin, D.J., Defanti, T.A., Kenyon, R.V., Hart, J.C.: The CAVE: Audio visual experience automatic virtual environment. Communications of the ACM 35(6), 64-72 (1992)

8. Slater, M., Steed, A., Chrysanthou, Y.: Computer Graphics and Virtual Environments. Addison Wesley, Reading (2002)

9. Applied Science Laboratories: Operation Manual Mobile Eye (January 2007)

10. Haffegee, A.: VieGen: An Accessible Toolset for the Configuration and Control of Virtual Environments. Ph.D thesis, University of Reading (March 2008) 